\title{
Design and Manufacture of the Superconducting Bus-Bars for the LHC Main Magnets
}

\author{
L. Belova, M. Genet, J.-L. Perinet-Marquet, P. Ivanov, and C. Urpin
}

\begin{abstract}
The main magnets of the LHC are series-connected electrically in different powering circuits by means of superconducting bus-bars, carrying a maximum current of $13 \mathrm{kA}$. These superconducting bus-bars consist of a superconducting cable thermally and electrically coupled to a copper profile all along the length. The function of the copper profile is essentially to provide an alternative path for the current in case the superconducting cable loses its superconducting state and returns to normal state because of a transient disturbance or of a normal zone propagation coming from the neighboring magnets. When a superconducting bus-bar quenches to normal state its temperature must always stay below a safe values of about $100{ }^{\circ} \mathrm{C}$ while the copper is conducting. When a resistive transition is detected, the protection systems triggers the ramping down of the current from $13000 \mathrm{~A}$ to 0 . The ramp rate must not exceed a maximum value to avoid the transition of magnets series-connected in the circuit.

This paper concerns the design and the manufacture of the high current superconducting bus-bars needed to interconnect the magnetic elements of the main dipoles, the main quadrupoles of the arcs and of the dispersion suppressors of the LHC.
\end{abstract}

Index Terms-Bus-bar, hollow-copper profile, thermal compensation joint.

\section{INTRODUCTION}

I NSIDE a magnet cold mass, three sets of superconducting bus-bars are present, one set for the dipole excitation, one for the focusing quadrupoles and one for the defocusing quadrupoles. They will be connected in the LHC tunnel to the corresponding sets of the neighboring cold masses.

All these sets of bus-bars are immersed in superfluid helium. The maximum length of a bus-bar is close to 17 meters. The total length of $13000 \mathrm{~A}$ rated current superconducting bus-bars of the entire LHC amounts about $150 \mathrm{~km}$.

\section{Bus-BAR MANufacturing PROCESS}

The standard bus-bar manufacturing process which involved brazing the superconducting cable onto an already-formed copper profile has been abandoned in favor of a bus-bar design that has the advantage of protecting the superconducting cable mechanically and thermally and having an approximately $50 \%$ lower labor cost. The saving is larger for the twisted and tortuous bus-bars such as the quadrupole ones.

This method consists in inserting the superconducting cable into a straight hollow copper profile, and after the profile has

Manuscript received September 24, 2001.

L. Belova and P. Ivanov are with the Budker Institute of Nuclear Physics, Novosibirsk.

M. Genet is with CERN, EST Division.

J.-L. Perinet-Marquet and C. Urpin are with CERN, LHC Division.

Publisher Item Identifier S 1051-8223(02)04185-4.
TABLE I

Bus-BAR CABLE CHARACTERISTICS

\begin{tabular}{ll} 
Width & $15.10+0,-0.02 \mathrm{~mm}$ \\
Mid-thickness at $50 \mathrm{Mpa}$ & $1.480 \pm 0.006 \mathrm{~mm}$ \\
Keystone angle & $0.90 \pm 0.05 \mathrm{deg}$ \\
$\begin{array}{l}\text { Transposition pitch } \\
\text { Number of SC strands }\end{array}$ & $100 \pm 5 \mathrm{~mm}$ \\
$\begin{array}{l}\text { Copper to superconductor } \\
\text { volume ratio }\end{array}$ & $1.95 \pm 0.05$ \\
Min. Icr at $1.9 \mathrm{~K}$ & $12960 \mathrm{~A}$ at $9 \mathrm{~T}$ \\
RRR & $\geq 70$ \\
\hline
\end{tabular}

been submitted to all the brazing and machining operations that might be harmful to the superconducting cable. Once the superconducting cable is inserted, the whole assembly undergoes successive bending and twisting operations.

According to a process described below, the gap inside the copper profile is then filled with a tin-silver solder to provide both thermal and electrical contact between the superconducting cable and the stabilizing copper. Each individual bus-bar is insulated with two layers of polyimide tape and a layer of polymerized fiberglass epoxy tape. An assembly of the insulated bus-bars inside fiberglass epoxy resin profiles provides a compact structure ready to be installed inside the cold mass.

\section{BUS-BAR ELEMENTS}

\section{A. Superconducting Cable}

All the high current superconducting bus-bars of the LHC are equipped with the same type of superconducting cable as used for the outer coil layer of the main dipoles and the quadrupoles. The main parameters of this cable are listed below in Table I.

\section{B. Copper Profile}

Since the copper cross-section area limits the temperature raise it is optimized according to the maximum current ramping down rate. There are two different cross-sections of hollow copper profiles: the cross-section A intended for interconnecting the main dipole coils whose ramping down rate does not exceed $120 \mathrm{~A} / \mathrm{s}$ and the cross-section B for interconnecting the main quadrupoles, whose maximum ramping down rate is $420 \mathrm{~A} / \mathrm{s}$. 
TABLE II

Hollow COPPER PROFILE CHARACTERISTICS

\begin{tabular}{ll} 
Copper grade & $\begin{array}{l}\text { Cu-OF, with oxygen content } \\
\text { below } 10 \mathrm{ppm} .\end{array}$ \\
Electrical resistivity & $\leq 1.72410^{-8} \Omega \cdot \mathrm{m}$ at $20^{\circ} \mathrm{C}$ \\
Residual resistivity ratio & $\geq 100$ \\
Hardness & $\leq 65 \mathrm{HV}$ \\
\hline
\end{tabular}

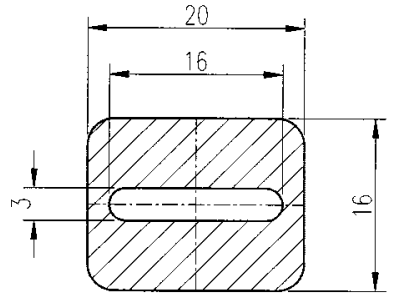

Cross section A area $269 \mathrm{~mm}^{2}$

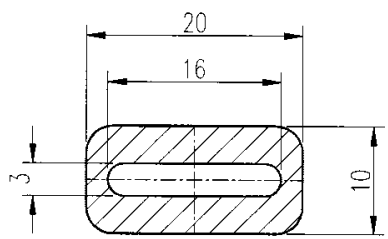

Cross section $B$ area $148 \mathrm{~mm}^{2}$
Fig. 1. Cross-sections of the hollow-copper profiles.

The hollow-profile copper grade is suitable for the various forming, soldering and welding operations necessary for bus-bar manufacture and shows a low residual electrical resistance at low temperature after completion of the bus-bar manufacture.

A high temperature annealing in a protective atmosphere is to be carried out to remove all oil traces from the profile inner surface in view of the superconducting cable soldering. This final annealing treatment offers two other advantages: on one hand it increases the residual resistance ratio of the copper profile and on the other hand it allows the conditioning of the profiles in flat pancake coils. This later is a substantial payoff, because it eases the handling of the 260 tons of hollow copper profiles required for LHC bus-bars.

\section{Thermal Compensation Joints}

The variations in the length of the busbars due to temperature differences of the order of $300 \mathrm{~K}$ are compensated by lyre-shaped thermal compensation joints hereafter referred to as lyres. All the busbars have been designed in such a way as to suffer no mechanical constraints, whatsoever the machine is cold and in operation. However, when the machine is heated up, the lyres are subjected to forces of compression. Fig. 2 shows the two types of lyres used for the main dipole bus-bars.

The lyres are an integral part of the bus-bars rather than added components. The continuity of the hole inside the copper profile containing the superconducting cable is preserved. The copper cross-section is not constant every where but is machined at the level of the lyres to reduce the copper thickness and thereby increase transverse flexibility in that region. To prevent an increase in the current density, this reduction in thickness is compensated by the addition of two packs of copper blades, diffu- sion-welded at the ends. These packs are welded onto each of the recessed parts of the copper profile.

The lyre is mechanically formed at the same time as all the other parts of the bus-bar, i.e., after the superconducting cable has been inserted.

\section{SofT-Soldering OF THE SuPERCONDUCTING CABLE}

Soft-soldering operation is carried out when the superconducting cable is inside its copper stabilizer and the process of mechanical forming, including the lyre-shaped part, has been completed.

Prior to inserting the superconducting cable, holes are drilled at each extremity of the busbar connecting the hole containing the superconducting cable to two receptacles, to be filled first with soldering flux and then the filler metal.

Filler metal is a silver-tin alloy (4\% $\mathrm{Ag}-96 \% \mathrm{Sn})$.

The orifice and the superconducting cable are rinsed using a halogen-free soldering flux and then the filler metal is introduced into one of the receptacles.

A water circulation device cools the extremities of the busbar thereby preventing the filler metal from escaping via the extremities. This end-cooling device remains in operation throughout the soldering and cooling processes.

The bus-bar and receptacles are heated up to the fusion temperature of the filler metal at a level of $230{ }^{\circ} \mathrm{C}$.

Once this temperature has been uniformly achieved, a compressed air at a pressure of 5 bars is applied to the receptacle containing the filler metal. After a short time, the filler metal surges up into the receptacle that was initially left empty.

The pressure on the first receptacle is released and applied to the second receptacle in order to reverse the direction of flow of the filler metal.

The pressure is then removed everywhere.

The whole busbar is gradually cooled down, starting from the center and spreading out to the extremities. A cooling rate of $10^{\circ} \mathrm{C}$ per minute with a temperature gradient of maximum $20^{\circ}$ per meter has proved to be highly satisfactory.

\section{INSULATION}

Bus-bar insulation must withstand test voltage of 4000 volts between the metallic part of the bus-bar and surroundings. This requirement has to be fulfilled in superfluid, gas or liquid helium for any pressure between vacuum and 20 bars and any temperature between $2 \mathrm{~K}$ and $400 \mathrm{~K}$. The insulation is permeable to superfluid helium. In case of a quench, insulation allows the evacuation of the helium gas without any degrading of the insulating materials.

\section{A. Plain Bus-Bar Insulation}

In order to fulfill the above requirements the bus-bars are insulated with two layers of polyimide tape followed by a pre-impregnated layer, with a $3 \mathrm{~mm}$ gap between turns. The two layers of polyimide tape without any glue are wound in opposite direction with an overlapping of 50\%. The pre-impregnated layer is wound in opposite direction to the outer polyimide one. This layer is cured at a temperature of $170{ }^{\circ} \mathrm{C}$. 


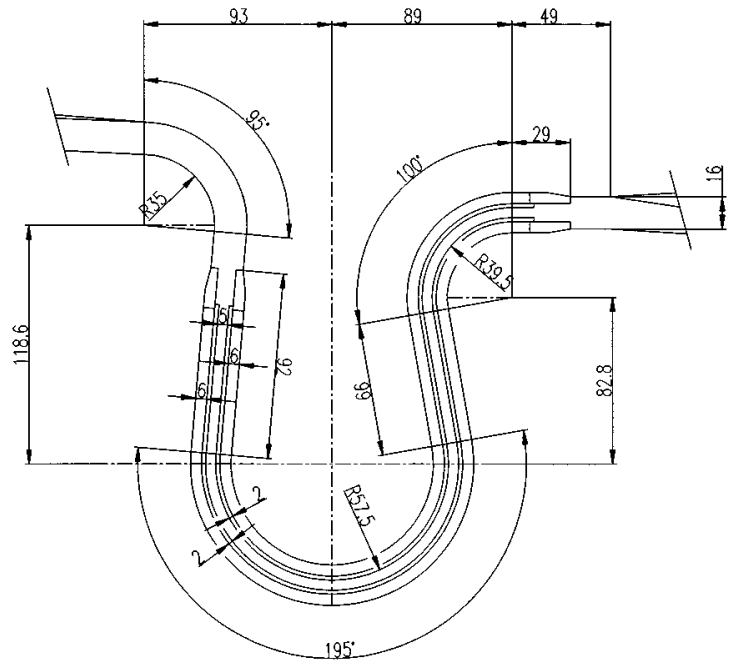

Thermal expansion joint with section A

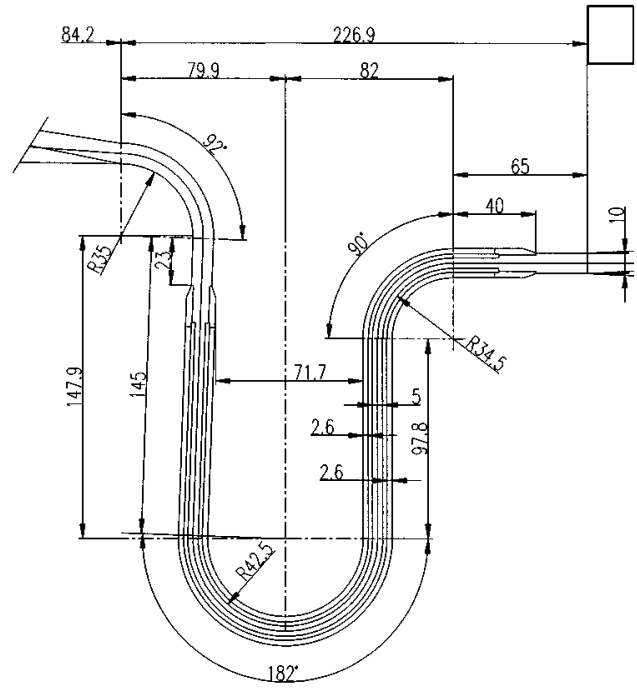

Thermal expansion joint with section $B$

Fig. 2. Schemes of LHC main dipole lyres.

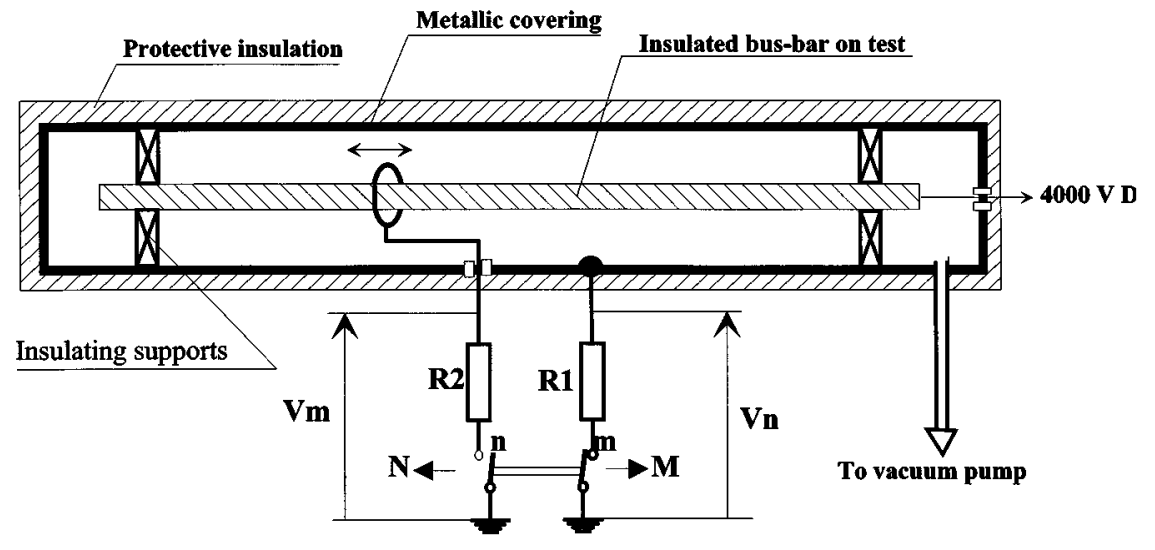

M switch position: detection of defect

N switch position: localization of defect

Fig. 3. Insulation test of the bus-bars.

\section{B. Lyres Insulation}

The two sets of copper blades and the central hollow copper conductor of the lyres are insulated separately with two layers of polyimide tape wound in opposite direction with overlapping of $50 \%$. To reduce the stiffness, the external pre-impregnated layer is replaced by rings of polymerized fiberglass tape to hold the two polyimide layers.

To increase safety, each lyre is embedded in a glass-cloth sleeve.

\section{Insulation Test of the Bus-Bars}

The dielectric strength of the insulation between the metallic part (stabilization copper-superconducting cable) and its outside environment is tested using $4000 \mathrm{~V} \mathrm{DC}$.

The insulated bus-bar is placed inside a metal container capable of withstanding an inside vacuum, see Fig. 3.
Inside this container, the bus-bar is put on nonconducting supports, its visible end conducting parts insulated.

The metallic part of the bus-bar is brought to $4000 \mathrm{~V}$ with respect to the conducting inner surface of the container, which is connected to the earth through a resistor.

Under these conditions a vacuum is gradually produced within the container. During this steady reduction in pressure from normal pressure to $30 \cdot 10^{-6}$ bar, the dielectric disruption potential of the residual atmosphere between the inner surface of the capsule and the bus-bar metallic area around the insulation defect, falls to a value much lower than the test voltage (Pachen's law).

Any insulation defects or channels between the insulation overlapping layers with characteristic dimensions of about 100 microns can be detected and located accurately by moving the earth connection along the bus-bar as shown on Fig. 3. Defect detection is carried out with switches in " $m$ " position 


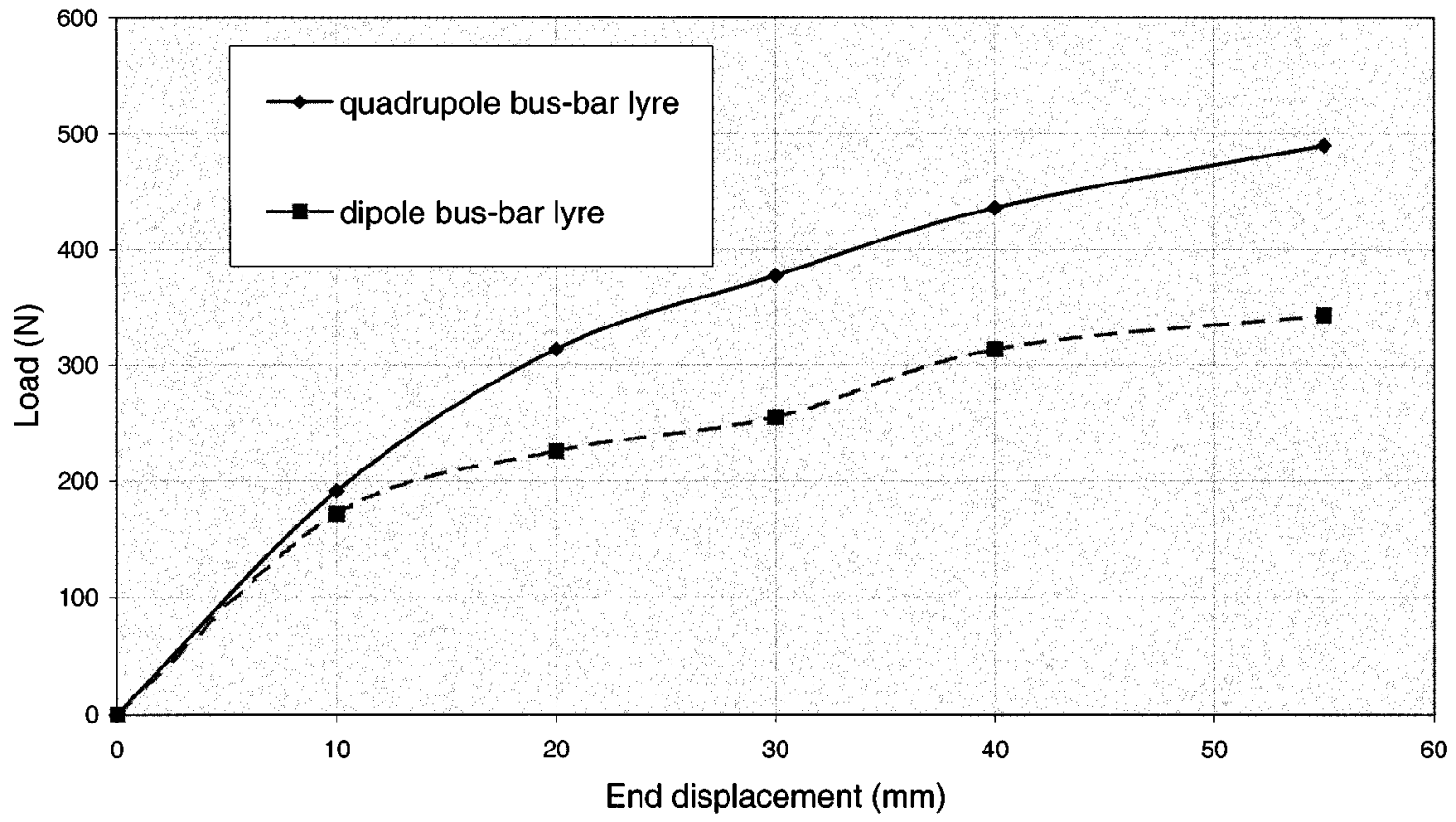

Fig. 4. Compressive load versus the length variation of the lyres of the main dipole magnets.

and defect location with switches in " $n$ " position by measuring respectively the voltage drop on the resistors $\mathrm{R} 1$ and $\mathrm{R} 2$.

This simple and reliable method is well adapted to seriesproduction.

\section{IMPACT ON PERFORMANCE OF THIS NEW METHOD}

A comparison of this new bus-bar manufacturing process with the traditional one mentioned in paragraph II results in:

\section{A. Improved Bus-Bar Performances}

Due to the central location of the superconducting cable within the copper profile, a better thermal coupling is achieved between the cable and the stabilizing copper. This implies higher cable stability with regard to external disturbances.

\section{B. Improved of Reliability}

When a bus-bar is bent the plane of zero mechanical deformation lies in the plane of the superconducting cable, hence there is less stress in the cable especially in the region of the lyres during warm-up and cool-down.

\section{PARAMETERS MEASURED}

\section{A. Measurement of the Bus-Bars' Residual Resistance Ratio}

The residual resistance ratio (RRR) was measured between the extremities of two bus-bars similar to those in the main LHC dipole, one made using cross-section A and the other cross-section B. The RRR values measured were between 210 and 220. The measured RRR of similar bus-bars produced using the solid conductor method was of the order of 70 .

This remarkable increase in the RRR is due first to the annealing treatment to which the profiles are submitted at the end of the manufacturing process and, second to the reduction in the

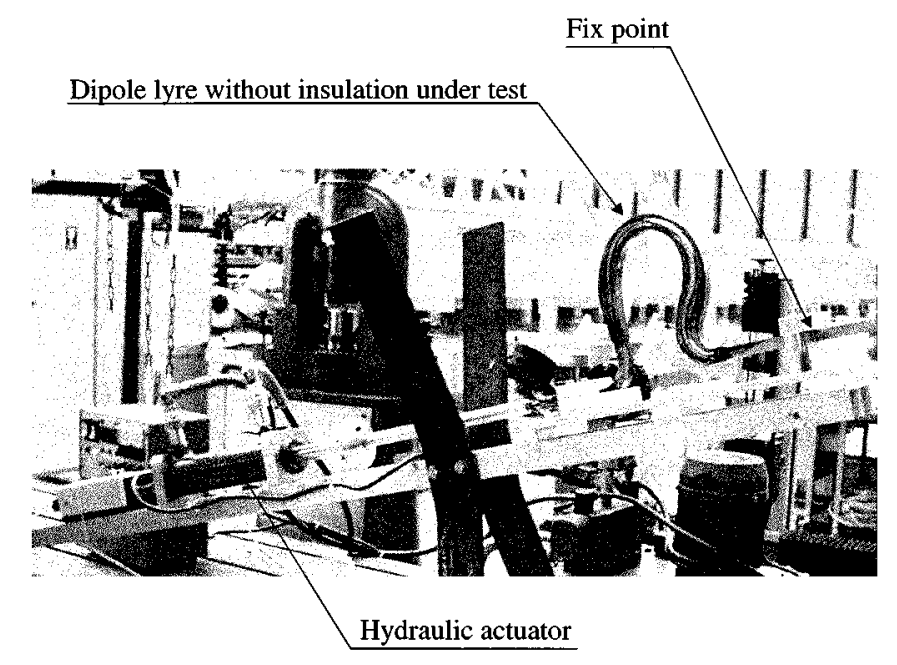

Fig. 5. Test equipment for endurance test of the lyres.

thermal constraints during the brazing of the superconducting cable inside the copper stabilizer. The mechanical constraints during the fabrication are identical, since they are connected with the longitudinal shape of the bus-bars.

\section{B. Longitudinal Rigidity of the Lyres}

Fig. 4 gives two typical measured curves of the compressive loads versus the length variations of the insulated lyres of a quadrupole and a dipole bus-bars of the main dipoles.

\section{Quantification of the Mechanical Reliability of the Lyres}

Destructive mechanical endurance tests have been performed on both types of the lyres of the main dipoles. Their extremities were submitted to compression cycles with an amplitude of $55 \mathrm{~mm}$ with respect to the rest position. Thermal contraction due to cooling down is $47 \mathrm{~mm}$. 
These tests were carried out both in liquid nitrogen with insulated lyres and at room temperature after removing the insulation for inspection of any damages.

In liquid nitrogen no deterioration was observed after 2000 cycles. The fatigue test of the lyre without insulation at room temperature, showed appearance of hairline cracks on the copper (observation by microscope) after 1500 more cycles for the dipole lyres and 2500 more cycles for the quadrupole lyres.

After each test the superconducting filaments of the cable located at the level of the lyres under load were systematically inpected after the copper was dissolved by nitric acid. None of the superconducting filaments were damaged or cut.

\section{Solder Filling Coefficient}

The solder filling coefficient, defined as the ratio of the mass of the solder inserted to the mass of solder required to fill entirely the hole of the profile with the superconducting cable inside, is higher than $95 \%$. This filling quality, very easy to obtain using fluxes containing corrosive halogens, can now be guaranteed using pure collophane-based fluxes.

\section{E. Operational Results of the Bus-Bars}

An experiment designed to quantify the operational parameters of the new-generation busbars is in progress. These tests will principally measure the heating up of the bus-bars after a quench and the velocity of the quench propagation along the bus-bars. These measurements will be carried out in superfluid, liquid, and gaseous helium.

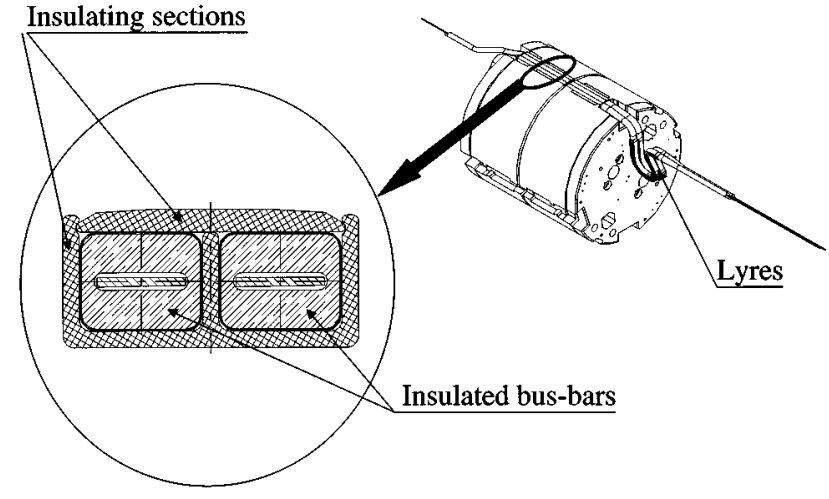

Fig. 6. Installation of dipole bus-bars inside a dipole.

\section{INSTALLATION OF THE BUS-BARS INSIDE THE Cold Masses}

Insulating profiles provide the housing for the superconducting bus-bars inside the cold masses of the LHC main magnets. Fig. 6 shows the installation of the main dipole bus-bars inside the dipole cold mass.

\section{CONCLUSION}

At the time of publication of this paper, production of main dipole bus-bar is starting successfully at Budker institute of Nuclear Physic in Novosibirsk.

\section{ACKNOWLEDGMENT}

The authors would like to extend special gratitude to C. Wyss and J. Vlogaert for constant attention to the project. 